\title{
SEAHORSE Project: Dealing with Maritime Workarounds and Developing Smarter Procedures
}

\author{
R.E. Kurt, V. Arslan \& O. Turan \\ Department of Naval Architecture, Ocean and Marine Engineering, University of Strathclyde, Glasgow, UK
}

L.D. Wolff \& B. Wood

CalMac Ferries Limited, Gourock, UK

O. Arslan \& T. Kececi

Dept. of Maritime Transportation and Management Engineering, Faculty of Maritime, Istanbul Technical

University, Tuzla-Istanbul, Turkey

J. W. Winkelman \& M.V. Wijngaarden

Jumbo Shipping, Schiedam, Netherlands

G. Papadakis

$A P \& A$ Ltd, London, UK

ABSTRACT: EU funded SEAHORSE project is developing a novel approach to improve maritime SOPs. SEAHORSE Project's 'Smart Procedures Concept' aims to develop a system to capture the behavioural adaptations that crew have developed in order to cope with operational demands and challenges. Initially, a detailed survey was conducted worldwide to capture seafarer's safety attitudes as well as the common workarounds. Then, these collected workarounds will be categorised through conducting risk and benefit analysis. Accordingly, safe workarounds will be approved as Standard Operating Procedures (SOPs), on the other hand, measures will be proposed to prevent reoccurrence of unsafe workarounds. This paper will present the proposed approach to deal with maritime workarounds and report the preliminary results of the analysis.

\section{BACKGROUND INFORMATION}

Maritime industry witnessed catastrophic accidents throughout years such as Herald of Enterprise, Costa Concordia and Deep Water Horizon. Human or organisational errors are highlighted as the main reasons of these accidents which caused fatalities, ship losses and environmental pollution. IMO (2002) stated that "Shipping is perhaps the most international of all the world's great industries and one of the most dangerous." Shipping companies are trying to address human errors in a reactive way and try to develop measures to avoid reoccurrence of those errors, which have quite big impact in maritime domain currently.

It was stated that maritime transport is 25 times riskier than air transport according to the accounts for deaths for every $100 \mathrm{~km}$ (Berg, 2013). In the same paper, it was stated that operation of ships are subject to full of regulations, procedures and guidelines, which are expected to be adhered to by crew and officers. However, in some situations, instruction given to the bridge team may not be appropriate and may constitute supervisory violations as leaders find it difficult to adapt their instructions to changing situations due to possible poor safety culture. Similarly, Debra et al. (2007) reports that coastal pilots in Australia and New Zeeland could not report hazards as much as they would like to due to the commercial pressure from the client shipping companies. A sur- vey conducted reveals that $71 \%$ of port pilots in New South Wales and $62 \%$ of port pilots in Western Australia agreed that commercial pressure forces pilots working outside established rules (Debra et al., 2007).

International Safety Management (ISM) code, introduced in 1998, is aimed to bring self-regulation to the maritime industry. ISM is criticised due to its bureaucratic nature as it forces seafarers to fill many forms and checklist resulting in seafarers' time and focus are taken away from working safely (Bhattacharya, 2012). ISM requires shipping companies to develop work procedures involving management of risks, maintenance of ships and equipment, emergency preparedness as well as reporting incidents, accidents and near misses while auditing the current systems. On the other hand, due to downsizing of workforce, seafarers are expected to be multitasking and work longer hours while having fixed and short term contracts. In the same paper, however, survey with managers indicated that most common cause for accidents at sea was seafarers' noncompliance with SMS indicating seafarers' apathy towards following procedures (Bhattacharya, 2012). On the other hand, seafarers claimed that it is not the SMS but their skills gained through their long experience helped them to maintain shipboard safety.

Three types of errors identified by Reason (Reason, 1990) can be listed as Skill Based Performance, Rule Based Performance and Knowledge Based Per- 
formance. Rule based performance is the riskiest of all since rule breaking is seen as the contributory factor in most of serious incidents/accidents (Skalle et al., 2014). Rule Based mistakes are done by the crew for a good reason as they do not intend to cause damage; people fail to apply the correct rule or procedure or expressed in another way, or implement an inappropriate rule and mostly caused by misjudging the problem (Skalle et al., 2014). In the same paper, following a procedure is classified as both rule based and knowledge base, and therefore requires a lot of judgement to adjust the procedure to specific situations.

Knudsen (2009) states that efforts to improve safety resulted in increasing volume of regulations, control and paper work such as checklist, workplace assessment and risk assessment, and many seafarers view that these demands on seafarers are imposed by people who do not understand anything about seafarer's life and work. Rule following in several cases is seen as counteracting work against the proper seamanship. This may be due to the not well developed rules/procedures. Since humans are logical actors, deliberate additions and subtractions may be conceded irrespective of perceived risks in order to satisfy employers' demand for efficiency and productivity (El-Ladan and Turan, 2012).

Due to the lack of standardisation between vessels and poorly designed Standard Operating procedures, measures introduced to eliminate the errors fail to sustain desired level of safety. Standardisation is described as the accumulation of the efforts to prevent failures which were revealed based on accident investigations and the aggregations of learnt lessons from these accidents (Bieder and Bourrier, 2013). In maritime industry, even equipment, layout and operating practices of two sister ships can be different. When crew members start working on another vessel of the company, they need to create their own checklists to perform their tasks and encounter with several problems due to aforementioned factors. Maritime and aviation sectors, who are the leaders of the transportation, are heavily relying on standardisation by utilising Standard Operating Procedures, to achieve the desired level of safety.

\section{STANDARD OPERATING PROCEDURES IN MARITIME}

Standard Operating procedures are designed to show best and qualified practices to seafarers to perform their tasks, but in reality, tasks may differ in every ship due to non-standardisation. An early Dutch investigation association analysed accidents with regards to loss containment in chemical industry and revealed that $50 \%$ of the accidents related to procedures and this $50 \%$ consist of no or unclear proce- dure $(10 \%)$, wrong procedures $(12 \%)$ and current procedures weren't followed properly $(28 \%)$.

The Standard operating procedures are claimed that they may not be compatible with real shipping operating environment and it created the well-known dilemma "work as imagined" vs "work as done". Work as imagined described as what designers, managers, regulators and authorities believe happens or should happen. Work as done refers what actually happens. Also all of the efforts to standardise the ship operation by SOPs are ended up with excessive amount of paperwork which is almost very demanding challenge for seafarers.

\section{SEAHORSE APPROACH}

SEAHORSE Project aims to investigate the human performance problems through focusing on the maritime workarounds. A "workaround" in the context of SEAHORSE Project is "the deliberate changes that the crew have applied to standard procedures and to the equipment on ships, due to practical and other needs". Therefore, a workaround can also be explained as a method of creating a temporary solution to a problem or limitation in a system when usual defined procedure is not working. By this definition it is necessary that these workarounds should be monitored and analysed with the aim of properly addressing the limitations in system. Delcore (2009) proposes that workarounds can be used as a useful source of ideas for the improvement of products and services, SEAHORSE Project is developing a systematic approach to capture and learn from the maritime workarounds in order to achieve safer and more effective maritime operations.

SEAHORSE Smart Procedure Methodology will compare predefined SOPs and maritime workarounds reported by crew members. Reported workaround will be assessed in terms of risk and benefits by 3 different experts after securing anonymity of the data. After analysis of the reported workaround by experts, a feedback form will be sent to crew members explaining whether workaround is safer than the actual SOP or workaround has crucial risk potential.

\section{QUESTIONNAIRES}

SEAHORSE Project initially aimed to capture the current maritime workarounds through application of anonymous surveys. The SEAHORSE Project' questionnaire consists of 3 main sections as shown in Figure 1. 


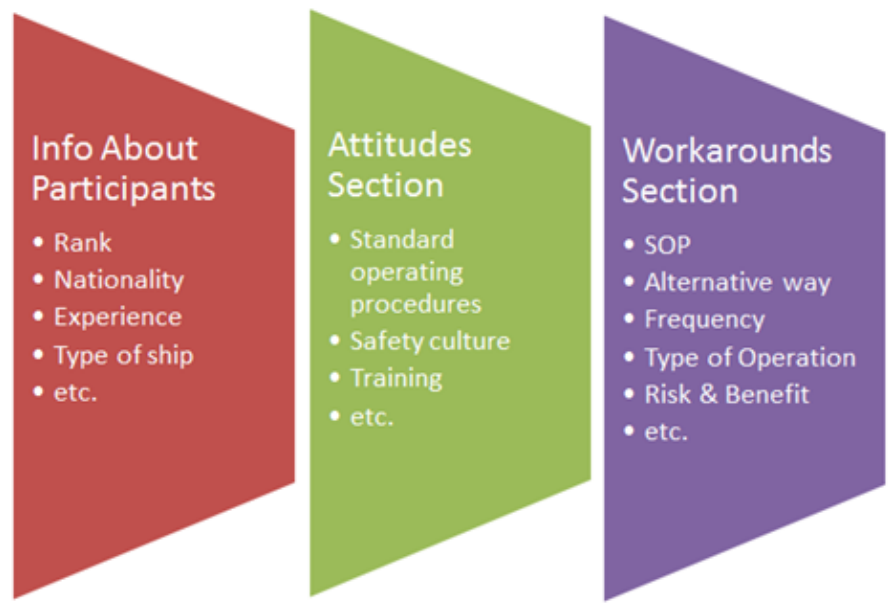

Figure 1. Sections of SEAHORSE Questionnaire

SEAHORSE Questionnaire is carefully developed with an interdisciplinary group to ensure that it captures the information, which is required to conduct further analysis (Questionnaire can be accessed via http://www.seahorseproject.eu/Questionnaire/tabid/4 608/Default.aspx).

The first section of the questionnaire aimed to collect demographic information about the participants such as: their role/rank on board, nationality and seagoing experience. However, it was ensured that anonymity of participants are protected, therefore, no personal information is collected which may lead to identification of the person. The second section focusses on capturing the attitudes of a seafarer where participants were asked to agree or disagree with the given statements by using a Likert Scale (i.e. never, rarely, some-times, often, and always). An example statement is shown below;

"Job tasks and related procedures required to be followed on ships are easy to follow"

In this section attitude statements were carefully selected and categorised in following sections; procedure design, training and competence, safety culture, employee - employer trust, matching procedures to operational reality. Third section is asked in an open-ended form which aims to capture the information related to workarounds as follows; description of workaround, related standard operating procedure (SOP), frequency, type of operation, underlying reason for the workaround.

Currently, returned questionnaires are being analysed and preliminary results are discussed in the following section.

\section{PRELIMINARY QUESTIONNAIRE RESULTS}

The aim of the SEAHORSE-Smart Procedure Survey is to capture general understanding of the seafarers towards safety. The questions are asked about seafarer's demography, attitudes and the workarounds that they performed. Totally, 451 numbers of responses are received about seafarers' attitude and 295 numbers of responses are received about maritime workarounds.

\subsection{Demographic Analysis}

The statistical analysis was performed by utilising SPSS to identify seafarer's ranks, overall seagoing experience, operation type, number of procedure they face on a daily basis. The majority of the seafarers are masters and chief officers with the percentage of $25 \%$ and $14 \%$ respectively. It presents participants of the surveys are quite expert on the bridge operations (Fig. 2).

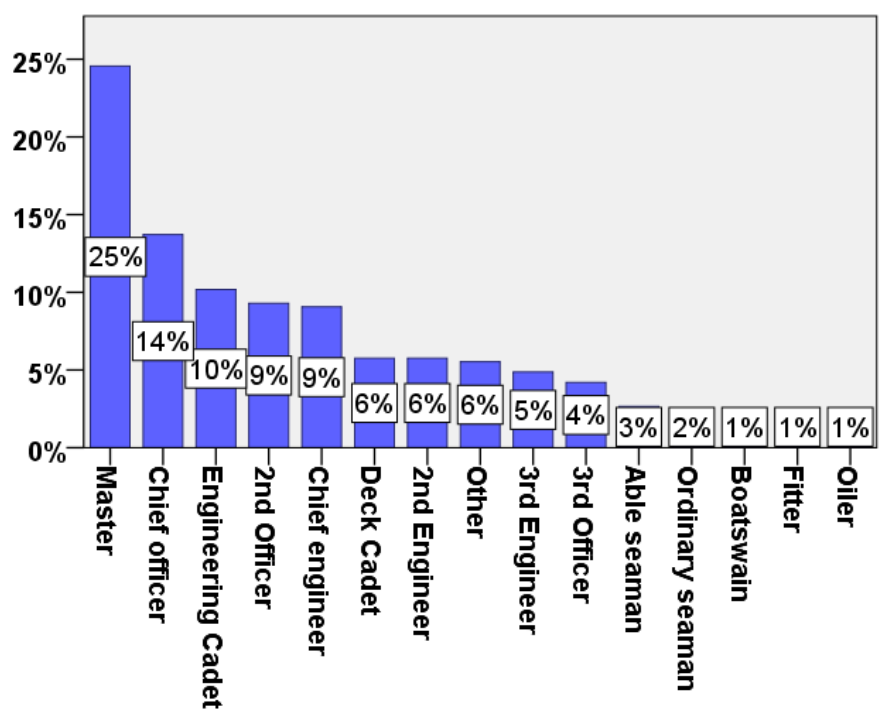

Figure 2. Distribution of participants' role on-board ships

The seagoing experience varies significantly among participants as it ranges from couple of months to 56 years. The average seagoing experience is found as 11.16 years with the standard deviation of 11.04 according to descriptive statistical analysis on SPSS. The overall seagoing experience distribution is given below in Figure 3.

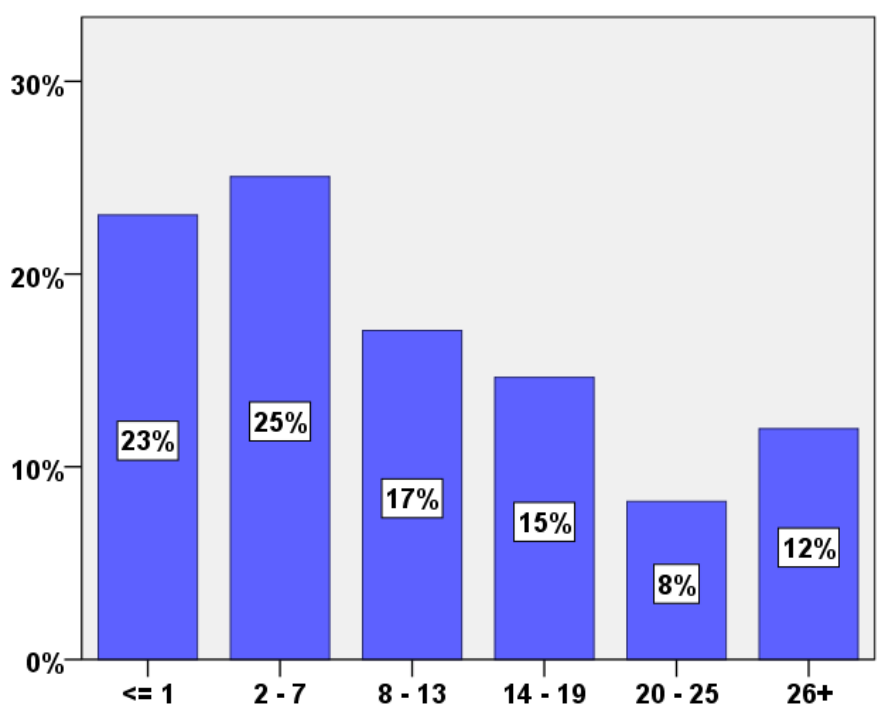

Figure 3. Overall seagoing experience distribution 
According to survey which was distributed in the world wide, majority of the seafarers work on tanker ships with the $31 \%$. It is followed by the dry cargo and other commercial vessels by $22 \%$ each (Fig. 4).

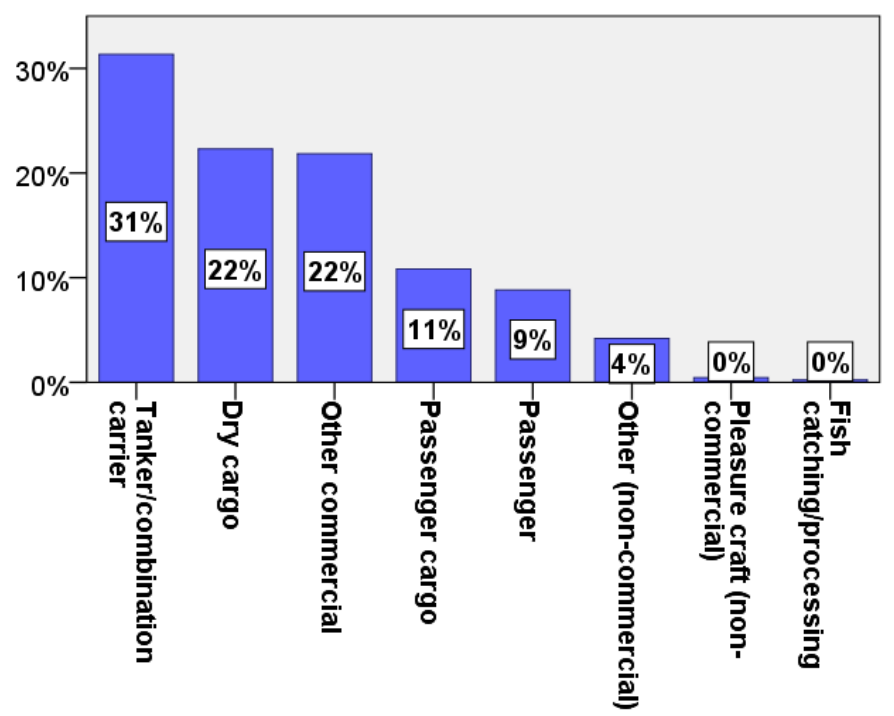

Figure 4. Vessel type

$54 \%$ of the seafarers work on deep-sea shipping companies (Fig. 5). Deep-sea shipping operation has ad hoc difficulties due to excessive number of operating days. This operation type was followed by shortsea shipping (35\%) and offshore shipping $(10 \%)$.

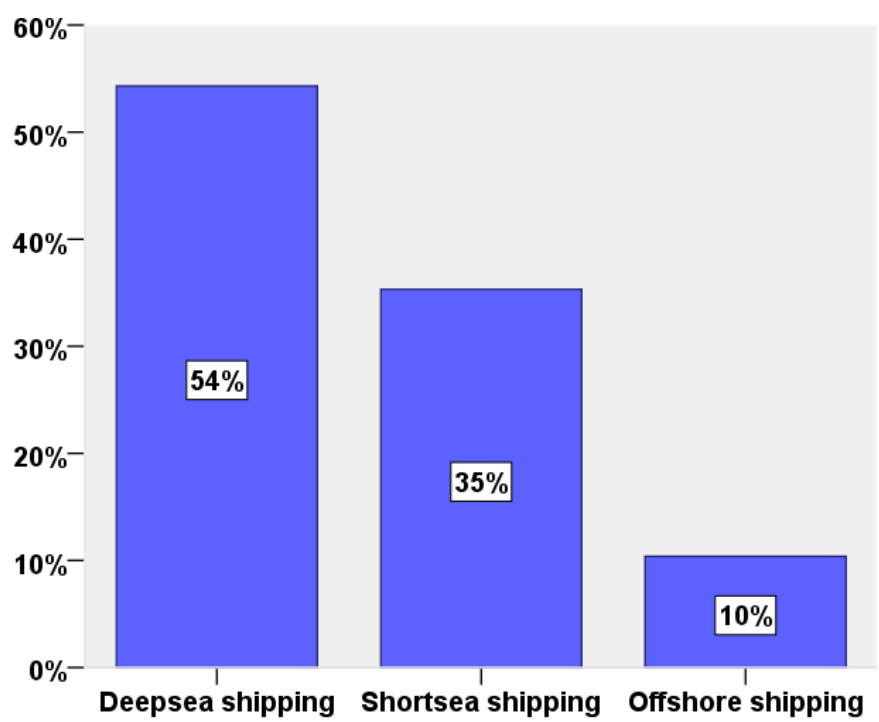

Figure 5. Operation type of shipping companies

Standard Operating Procedures are designed to provide the best way of working for seafarers and according to the type of operation, seafarers need to deal with number of tasks by following the relevant procedures on a daily basis. $31 \%$ of seafarers deal with up to 5 SOP per day while $20 \%$ of the seafarers deal with $15+$ operating procedures (Fig. 6).

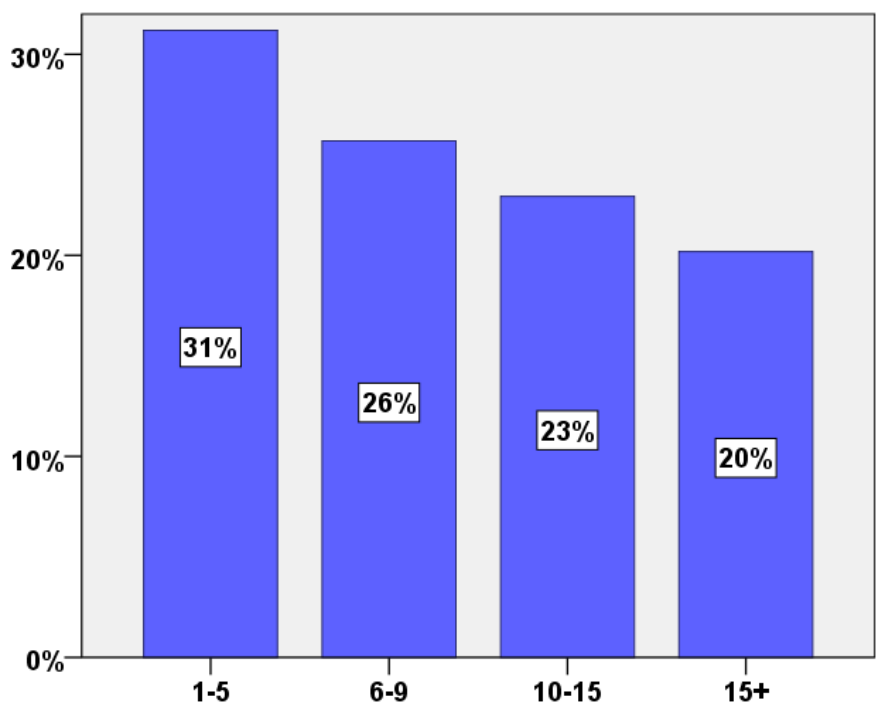

Figure 6. Number of operating procedures

\subsection{Attitudes Results}

In the attitude section of the questionnaire, the intention was to measure the safety climate in the company. In total 48 attitude questions were asked and questionnaire was structured as below:

- Procedure Design

- Training, competence

- Safety Culture

- Employee - Employer Trust

- Matching Procedures to Operational Reality

Each heading has several statements to capture the general views of seafarers about that specific area. There is a still big debate on what extend SOPs reflect the operational realities. Seafarers stated that majority of Standard Operating Procedures are factually incorrect. Also, seafarers are doubtful if really SOPs reflect best way of working or even they make the operations less efficient as described in Figure 7 and Figure 8.

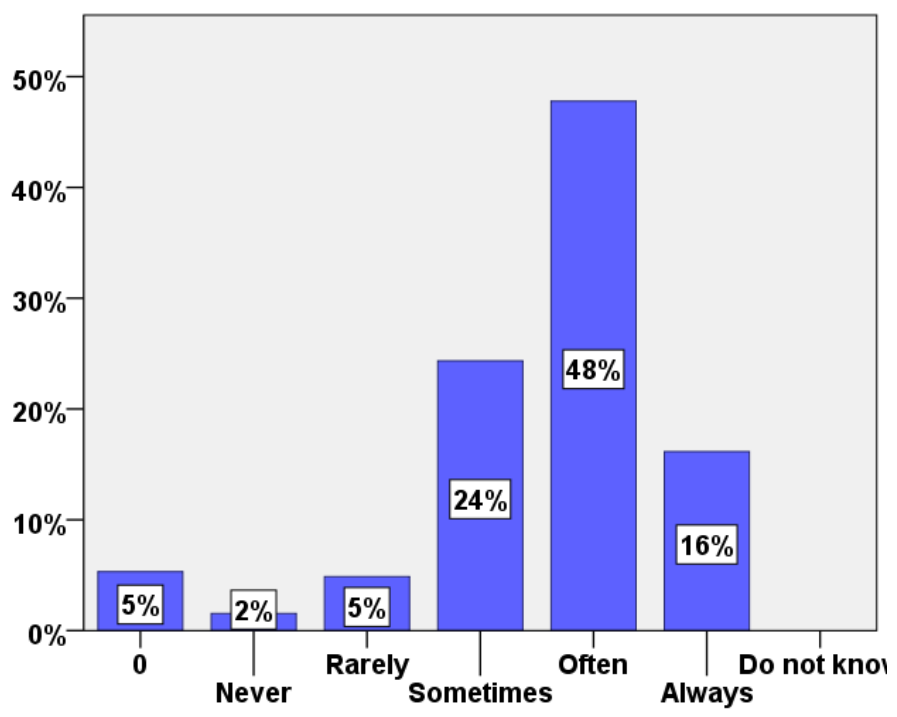

Figure 7. Statement: Job tasks and related procedures required to be followed on ships are factually incorrect 


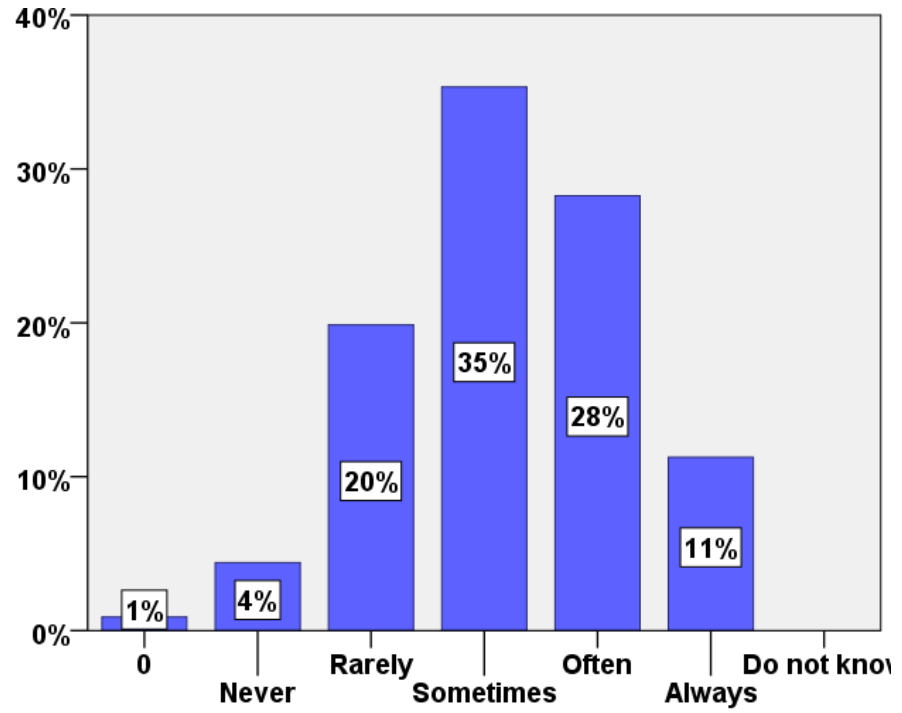

Figure 8. Statement: Some procedures that crew need to follow as part of their job tasks make the job less efficient

Significant efforts are invested to enhance competency of the crew members with appropriate and continues trainings by shipping companies to sustain desired level of safety. However, seafarers stated in the survey that they are not always trained on how to deal with unusual conditions (Fig. 9).

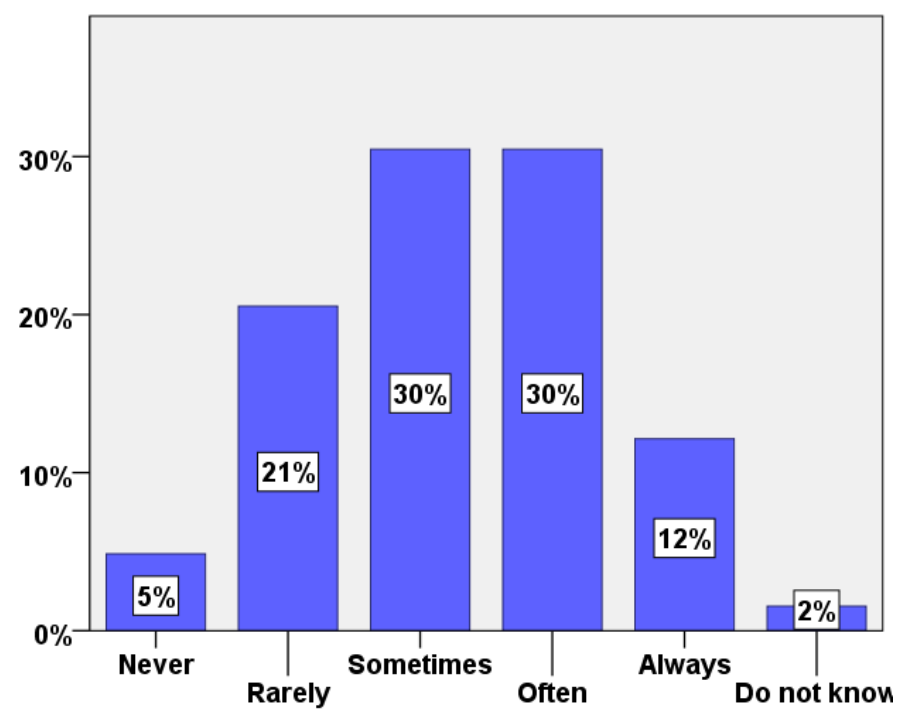

Figure 9. Statement: In our company crew members are trained on how to deal with unusual (infrequent) conditions (e.g. unexpected circumstances which are not defined in the procedures and guidelines).

SOPs are introduced to maritime domain for every single task and for every crew member from different rank but many of them ignore following them and adopt their own alternative way due to many reasons. Figure 10 illustrates that $37 \%$ of the crew sometimes follow the defined SOPs.

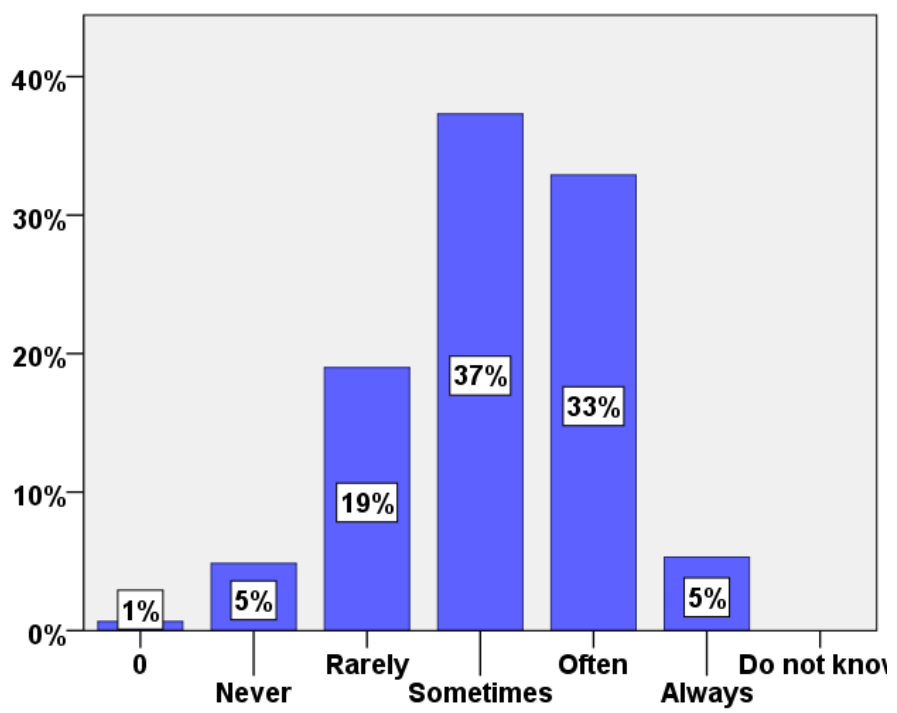

Figure 10. Statement: In shipping companies, it is common that procedures are not always followed.

In order to establish a good employer - employee trust, there should be anonymous reporting system where crew member can report impractical SOPs to avoid possible risks to person, ship and environment. Figure 11 indicates that only $26 \%$ of the companies have this system in their SMS.

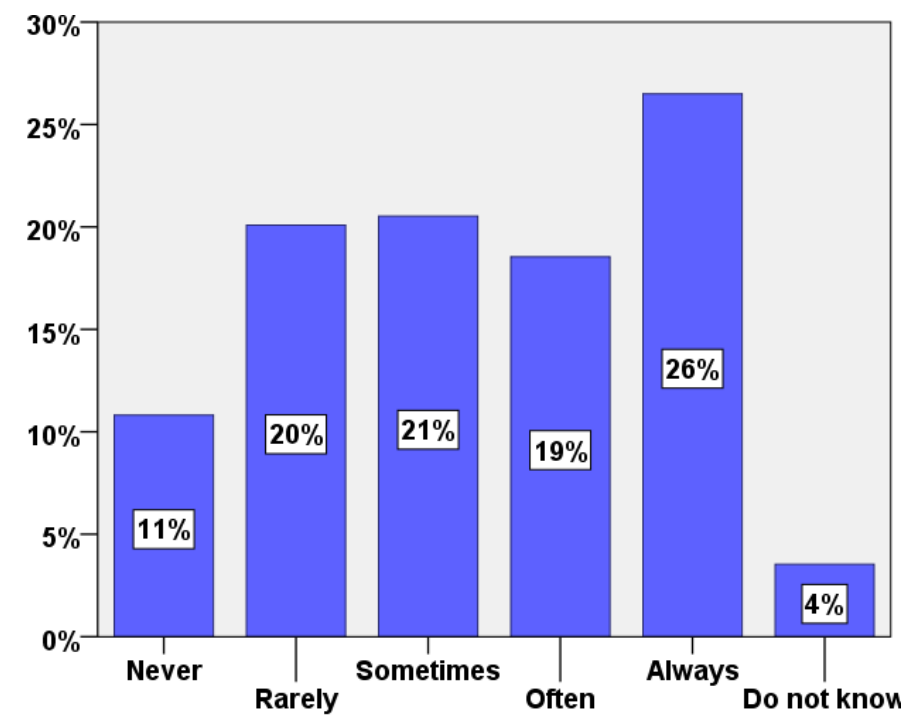

Figure 11. Statement: In our company, there are systems on board which allow crew members to report impracticable procedures anonymously.

Personnel Protective Equipment is extremely important for maritime operations to protect human from dangerous situations. Figure 12 shows that most of the people determined that they use PPE when it is required but workaround survey results show that ignoring the use of PPEs are very common in maritime domain. 


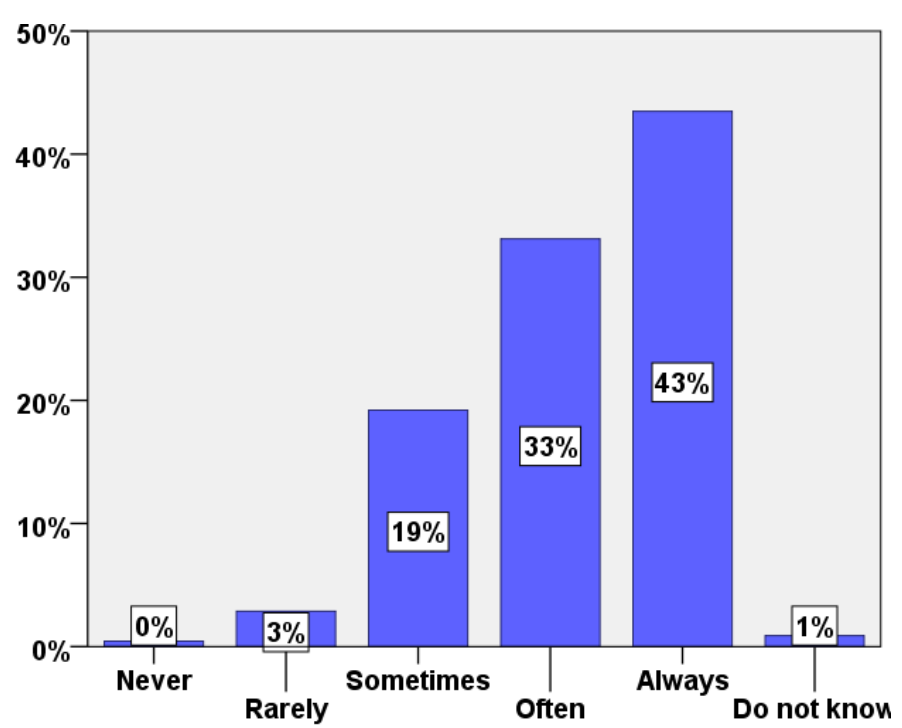

Figure 12. Statement: The members of our crew, use personal protective equipment when required.

\subsection{Workaround Results}

There is a common understanding in maritime domain that the shipping is highly regulated and seafarer needs to deal with numerous standard operating procedures on board ships. Due to high workload on board ship, seafarers sometimes develop their own approach to perform their tasks instead of strictly following SOPs.

In our questionnaire, 295 seafarers provided data about SOPs about a specific task and the alternative way to achieve their goals. Also they provided information about where they adopt this alternative procedure, what type of operations it is, what are the benefits of the following alternative way and whether this workaround is well accepted or not.

According to collected data from seafarers worldwide, the most frequent types of workarounds are identified as below:

- Paperwork ( details provided as an example case)

- Personal Safety Equipment

- Work-Rest Hours

- Navigational Standards \& Rules

- Hot-work \& permit to work

\section{Case:}

Paperwork is very well known issue in maritime domain. Most of the seafarers complain about that they can't perform their actual task due to excessive amount of paperwork. Danish Maritime Authority, (2013) stated that "When it comes to paperwork, most seafarers think that there is too much paperwork and documentation involved in many tasks, and that this takes up too much time and energy"

The example below indicates a very well-known workarounds with regards to paperwork
Definition of SOP: Prior to port arrival, and as sole navigator on the bridge, I spend most of my time in the chart room completing pre-arrival checklists and fixing the ship's position on paper charts. Many of the items on the checklists are either repeated or non-applicable. Ironically, as we move closer to the coast and to busier waters frequency of the fixing position is increased to align with the procedure. However, this distracts my attention from my main role of physically navigating the ship, it reduces spatial awareness. Procedure states that I can call the Master if workload becomes excessive, but due to limited manning I know he needs rest and such a call would be unwelcome. As an employee I'm expected to deal with it.

Definition of Alternative Way: Some crew completes the checklists after arrival, thus defeating their purpose. Some crew members ignore position fixing requirements and complete it at a more comfortable frequency.

Prevalence: Some of the crew members follow the same alternative practical way

Type of Operation: Bridge operation

Location: Navigation/communication control space

Reasons: Time constraint, need to focus attention to sailing the ship.

Benefits: Attention is focused on sailing the ship; this is fundamentally the most important aspect regarding the goal of getting the ship safely to port.

Risks: One may overlook important checks; trusting electronic means of monitoring the ship's position removes a valid safety barrier.

Participants of the survey asserted that all of these workarounds adopted by most of other crew members as well with the percentage of 37 (Fig. 13).

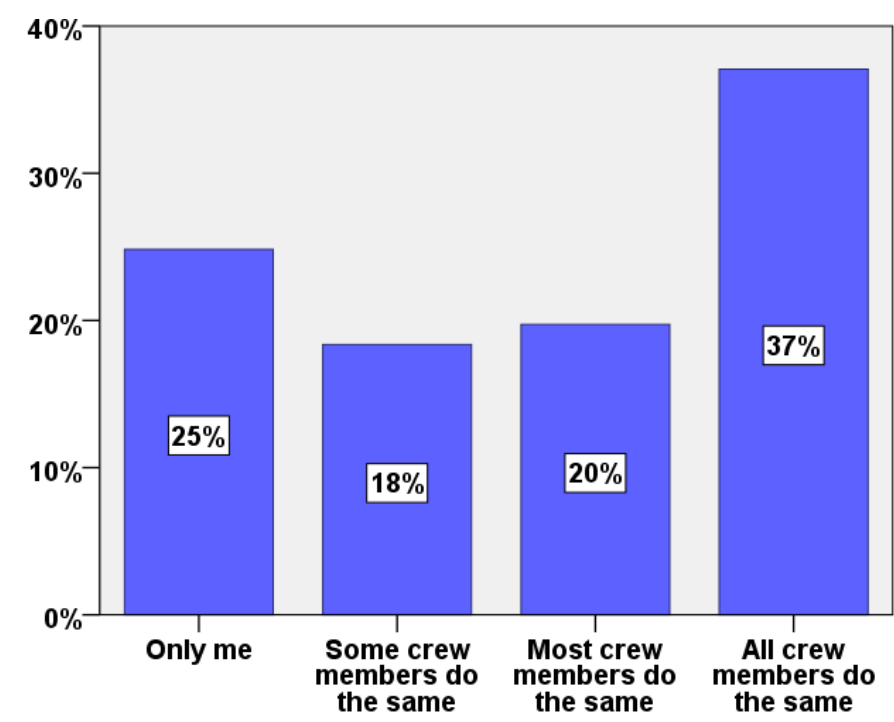

Figure 13. Statement: Please select the statement which suits best to the alternative practical procedure that you have defined in (b) 
The majority of the workarounds is held in deck operation (42\%) and followed by the engine room operation and bridge operation by $29 \%$ and $21 \%$, respectively (Fig. 14).

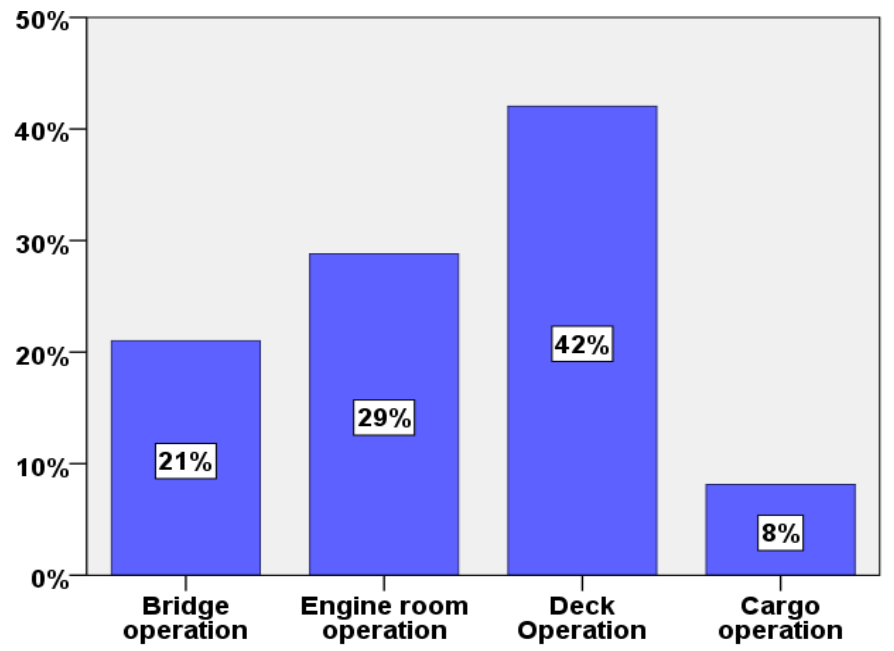

Figure 14. Types of operation

$21 \%$ of the workarounds are performed at the engine room (Fig. 15). It was followed by complete vessel and navigation/communication on control space with the $17 \%$ and $11 \%$ respectively.

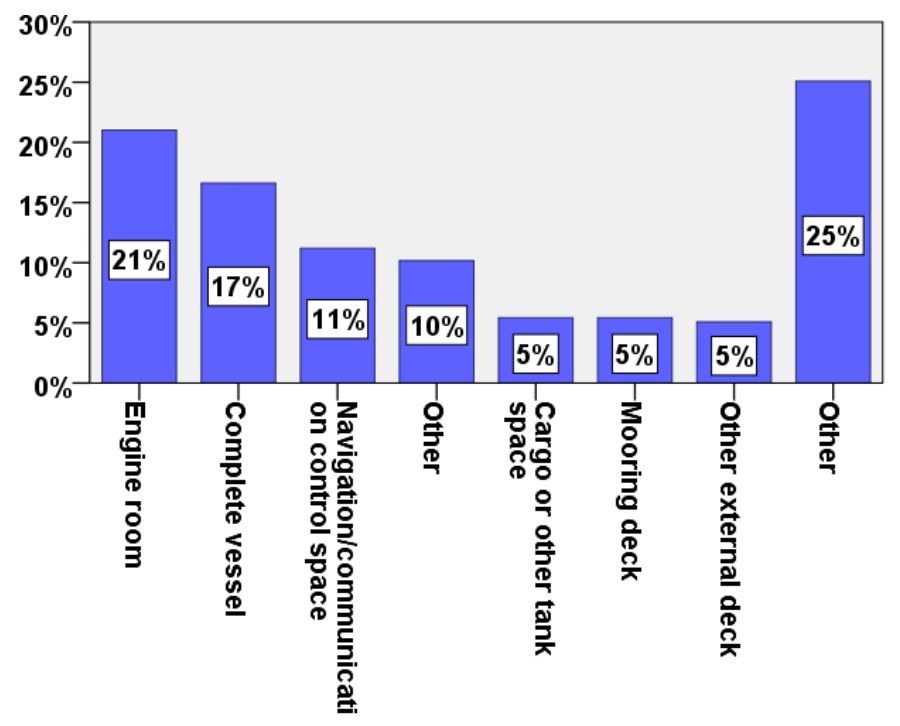

Figure 15. Location of workarounds

\section{FUTURE WORK}

Risk \& benefit analysis will be performed to compare all of these identified maritime workarounds and SOPs. The results is expected to indicate which way is safer and better. Good practices will be promoted and will turn into new Standard Operating Procedures. New actions will be developed to avoid reoccurrence of the bad practices.

\section{CONCLUSION}

Standard Operating Procedures are developed to generate best and safest practices in the maritime in- dustry but study showed that crew members adapt their own alternative ways to perform their tasks. Even though some workarounds are unacceptable in terms of safety, the analysis of maritime workaround revealed that high numbers of SOPs are not well designed. They do not reflect the maritime operational reality and they are ambiguous or impossible to follow on board ships. It was observed that sometimes seafarers couldn't explain SOPs of the tasks properly in the survey, this indicates that seafarers sometimes fail to understand actually what SOPs mean.

It was revealed that there are some workarounds which are conducted for real reasons however the chosen workaround is not the best way to address the problem. Also some of the crew members thought that their workaround was beneficial but actually the performed workaround breaches the international laws.

Finally, all of performed workarounds show that the maritime industry still have issues with regards to safety. Good practices are required to turn them into procedure while bad practices should be eliminated. Sometimes the reason why they perform this workaround is more important than the workaround itself.

\section{REFERENCES}

Berg, H.P. 2013. Human Factors and Safety Culture in Maritime Safety. The International Journal of Marine Navigation and Safety of Sea Transportation 7(3).

Bhattacharya, S. 2012. The effectiveness of the ISM Code: A qualitative enquiry. Marine Policy 36: 528-535.

Bieder, M.C. \& Bourrier, M. 2013. Trapping safety into rules: How desirable or avoidable is proceduralization? Ashgate Publishing, Ltd.

Danish Maritime Authority. 2013. Survey on Administrative Burdens among International Seafarers, Final Report - International Seafarers.

Darbra, R.M., Crawford, J.F.E., Haley, C.W., Morrison, R.J. 2007. Safety Culture and hazard risk perception of Australian and New Zeeland maritime pilots. Marine Policy 31: 736-745.

Delcore, H. 2009. Can't you just ask people? The Anthro Guys. Retrieved 08-08-2014 Source: http://theanthroguys.com /2009/09/17/can\%E2\%80\%99t-you-just-ask-people/.

Dimitrova, D.N. 2010. Seafarers' rights in the globalized maritime industry 75, Kluwer Law International.

El-Ladan, S.B. \& Turan, O. 2012. Human reliability analysisTaxonomy and praxes of human entropy boundary conditions for marine and offshore applications, Journal of Reliability Engineering and System Safety 98: 43-54.

IMO. 2002. Maritime Safety. Retrieved 06-08-2014 Source: http://www.imo.org/OurWork/Safety/Pages/Default.aspx.

Knudsen, F. 2009. Paperwork at service of safety? Workers' reluctance against written procedures exemplified by the concept of seamanship, Safety Science 47: 295-303.

Reason, J. 1990. Human Error, Cambridge University Press, Cambridge.

Skalle, P., Aomodt, A., Laumann, K. 2014. Integrating human related errors with technical errors to determine causes behind offshore accidents, Safety Science 63: 179-190. 